\title{
An evaluation of the effectiveness of a multi-modal intervention in frail and pre-frail older people with type 2 diabetes - the MID-Frail study: study protocol for a randomised controlled trial
}

\author{
Leocadio Rodríguez-Mañas ${ }^{1}$, Antony J Bayer ${ }^{3}$, Mark Kelly ${ }^{4}$, Andrej Zeyfang ${ }^{5}$, Mikel Izquierdo ${ }^{6}$, Olga Laosa ${ }^{2}$, \\ Timothy C Hardman, Alan J Sinclair ${ }^{8^{*}}$, on behalf of the MID-Frail Consortium
}

\begin{abstract}
Background: Diabetes, a highly prevalent, chronic disease, is associated with increasing frailty and functional decline in older people, with concomitant personal, social, and public health implications. We describe the rationale and methods of the multi-modal intervention in diabetes in frailty (MID-Frail) study.

Methods/Design: The MID-Frail study is an open, randomised, multicentre study, with random allocation by clusters (each trial site) to a usual care group or an intervention group. A total of 1,718 subjects will be randomised with each site enrolling on average 14 or 15 subjects. The primary objective of the study is to evaluate, in comparison with usual clinical practice, the effectiveness of a multi-modal intervention (specific clinical targets, education, diet, and resistance training exercise) in frail and pre-frail subjects aged $\geq 70$ years with type 2 diabetes in terms of the difference in function 2 years post-randomisation. Difference in function will be measured by changes in a summary ordinal score on the short physical performance battery (SPPB) of at least one point. Secondary outcomes include daily activities, economic evaluation, and quality of life.
\end{abstract}

Discussion: The MID-Frail study will provide evidence on the clinical, functional, social, and economic impact of a multi-modal approach in frail and pre-frail older people with type 2 diabetes.

Trial registration: ClinicalTrials.gov: NCT01654341.

Keywords: Multi-modal intervention, Frail, Pre-frail, Type 2 diabetes

\section{Background}

Diabetes has a high prevalence in ageing populations, affecting approximately $20 \%$ of people aged 70 years or over. It is anticipated that by 2050 the number of cases of diabetes will have increased by fourfold in people older than 70 years [1]. Diabetes is associated with increasing frailty and functional decline in older people [2]. Frailty is defined as a clinical syndrome in which three or more of the following criteria are present: unintentional weight

\footnotetext{
* Correspondence: alan.sinclair@beds.ac.uk

${ }^{8}$ Institute of Diabetes for Older People (IDOP), University of Bedfordshire, Putteridge Bury Campus, Hitchin Road, Luton, Bedfordshire LU2 8LE, UK Full list of author information is available at the end of the article
}

loss ( $\geq 4.5 \mathrm{~kg}$ in past year), self-reported exhaustion, weakness (grip strength), slow walking speed, and low physical activity [3].

Diabetes has serious personal and social consequences, and is a significant public health burden in terms of rising health care costs; in Spain, annual direct health care costs have been estimated at 2.5 billion euros [4]. In recent studies of older people, up to $28 \%$ of those with diabetes required some help with activities of daily living, compared with $16 \%$ of those without the condition [5]. This functional decline can be explained in only half of the cases by the classical complications of the disease, such as coronary artery disease, stroke, and peripheral vascular 
disease [5]. The worsening in functional status due to deterioration of skeletal muscle, increased co-morbidities, and adverse effects of overmedication associated with diabetes, results in many older frail people becoming more disabled, with an impaired quality of life associated with increased use of health care resources [6-8].

There is a marked lack of intervention studies that aim to reduce functional decline and improve quality of life in older people with diabetes and until relatively recently, most of the clinical guidelines for treating type 2 diabetes were of limited use in these subjects. A focus on improvements in function and well-being may be fundamentally of more clinical benefit in older frail people with diabetes than attention to metabolic control alone [9]. In older adults and frail individuals, resistance training is now considered to be an important component of diabetes management and prevention, mainly through increasing muscle mass, strength, and power [10]. This leads, in turn, to increased mobility and a decreased risk of falling [11]. In addition, resistance training has been proven to improve insulin sensitivity and fasting glycaemia, and to decrease abdominal fat in older people with type 2 diabetes [10]. It has been previously shown that intensive glucose lowering therapy (targeting a glycated haemoglobin level below 6.0\%) in patients with type 2 diabetes was associated with a reduction in 5-year nonfatal myocardial infarction but increased 5-year mortality $[12,13]$. More recently, it was reported that an intensive lifestyle intervention focusing on weight loss in overweight or obese adults with type 2 diabetes led to improvements in glycaemic control, blood pressure, high-density lipoprotein (HDL)-cholesterol, and triglycerides [14]. However, the intervention failed to reduce the incidence of cardiovascular events [15]. These observations provide sufficient motivation for evaluating non-metabolic control as an alternative (and complementary) way of improving clinical outcome.

The long-term impact of diabetes in an ageing population results in substantial health care expenditure. This further emphasises the potential value of an intervention that can prevent or delay the onset of diabetes-associated frailty. The multi-modal intervention in diabetes in frailty (MID-Frail) study focuses on the use of interventions designed to improve functional status and enhance quality of life by acting on the mechanisms involved in frailty and its progression to adverse outcomes [16]. The MID-Frail study brings together 16 partners from seven countries in the European Union (EU) and will run for 4 years, with the intervention lasting 2 years. The primary objective of the MID-Frail study is to evaluate, in comparison with usual clinical practice, the effectiveness of a multi-modal intervention (specific clinical targets, education, diet, and exercise) in frail and pre-frail subjects aged $\geq 70$ years with type 2 diabetes in terms of the difference in function 2 years post-randomisation. Difference in function will be measured by changes in summary ordinal score on the short physical performance battery (SPPB) of at least one point.

\section{Methods/design \\ Subjects \\ Inclusion criteria}

Subjects are eligible to enter the study if all of the following apply:

1) The subject is willing and able to give written informed consent for participation in the study.

2) The subject is aged 70 years or older, with a diagnosis of type 2 diabetes for at least 2 years.

3) The subject fulfils the Fried's criteria for frail or pre-frail individuals (Table 1 ).

4) The caregiver has agreed to participate in the study and to give informed consent for participation in the carer burden part of the study. If the caregiver does not agree, the subject may continue to participate in the study, but information about caregiver burden will not be collected.

Fried's criteria for frailty were chosen as they are easy to implement and allow the selection of subjects who are not dependent (moderately or highly). In addition, these criteria should avoid selection bias and prevent the enrolment of a high percentage of subjects with disabilities at baseline.

\section{Exclusion criteria}

Subjects cannot enter the study if any of the following apply:

1) Barthel score lower than 60 points [17].

2) Inability to complete the SPPB (total score $=0$ ) (Figure 1) [18].

3) Mini Mental State Examination (MMSE) score of less than 20 points [19].

4) Subject is unwilling or unable to consent or unable to participate safely in the intervention programme.

5) Previous history of myocardial infarction within 6 months, unstable angina, or congestive heart failure at stage III to IV of the New York Heart Association (NYHA) classification [20].

6) The subject is clinically unstable in the clinical judgment of the investigator.

7) Terminal illness (life expectancy $<6$ months).

8) Any other condition that, in the clinical judgment of the investigator, means that it would not be in the subject's best interests to enter the study.

9) Concurrent participation in a clinical trial or any other investigational study. 
Table 1 Fried's criteria for study inclusion

\begin{tabular}{|c|c|}
\hline Criterion & Definition \\
\hline $\begin{array}{l}\text { 1. Weight } \\
\text { loss }\end{array}$ & $\begin{array}{l}\text { Unintentional weight loss of } 4.5 \mathrm{~kg} \text { during the } \\
\text { past year }\end{array}$ \\
\hline 2. Exhaustion & $\begin{array}{l}\text { Using the responses (yes/no) to two statements on } \\
\text { the CES-D scale }\end{array}$ \\
\hline $\begin{array}{l}\text { 3. Physical } \\
\text { activity }\end{array}$ & $\begin{array}{l}\text { Is the weekly physical activity of the subject lower } \\
\text { or equal to (yes/no): } \\
\text { men: }<383 \text { kcal per week (walking: }<2.5 \text { hours per week) } \\
\text { women: }<270 \text { kcal per week (walking }<2 \text { hours per week) }\end{array}$ \\
\hline 4. Slowness & $\begin{array}{l}\text { Assessed by walk time and stratified by gender } \\
\text { and height }\end{array}$ \\
\hline 5. Weakness & $\begin{array}{l}\text { Assessed by grip strength and stratified by gender } \\
\text { and BMI }\end{array}$ \\
\hline
\end{tabular}

Frailty is indicated by satisfying three or more of the criteria; pre-frailty is indicated by satisfying one or two of the criteria [3]. BMI, body mass index; CES-D, Center for Epidemiologic Studies Depression.
To date, the study has been approved by 16 institutional ethics committees in Spain (see further details in Additional file 1). The study will be carried out in accordance with Good Clinical Practice, applicable local regulatory requirements, and the guiding principles of the Declaration of Helsinki.

\section{Study design}

This is an open, randomised, multicentre study, with random allocation by clusters (each trial site) to a usual care group or an intervention group (Figure 2). Each site will enroll on average 14 or 15 subjects. National research centres in Belgium, Czech Republic, Italy, and Germany (one each) and in France, United Kingdom, and Spain (two each) will each be responsible for 11 or 12 sites. Each site will be monitored to ensure full adherence to the
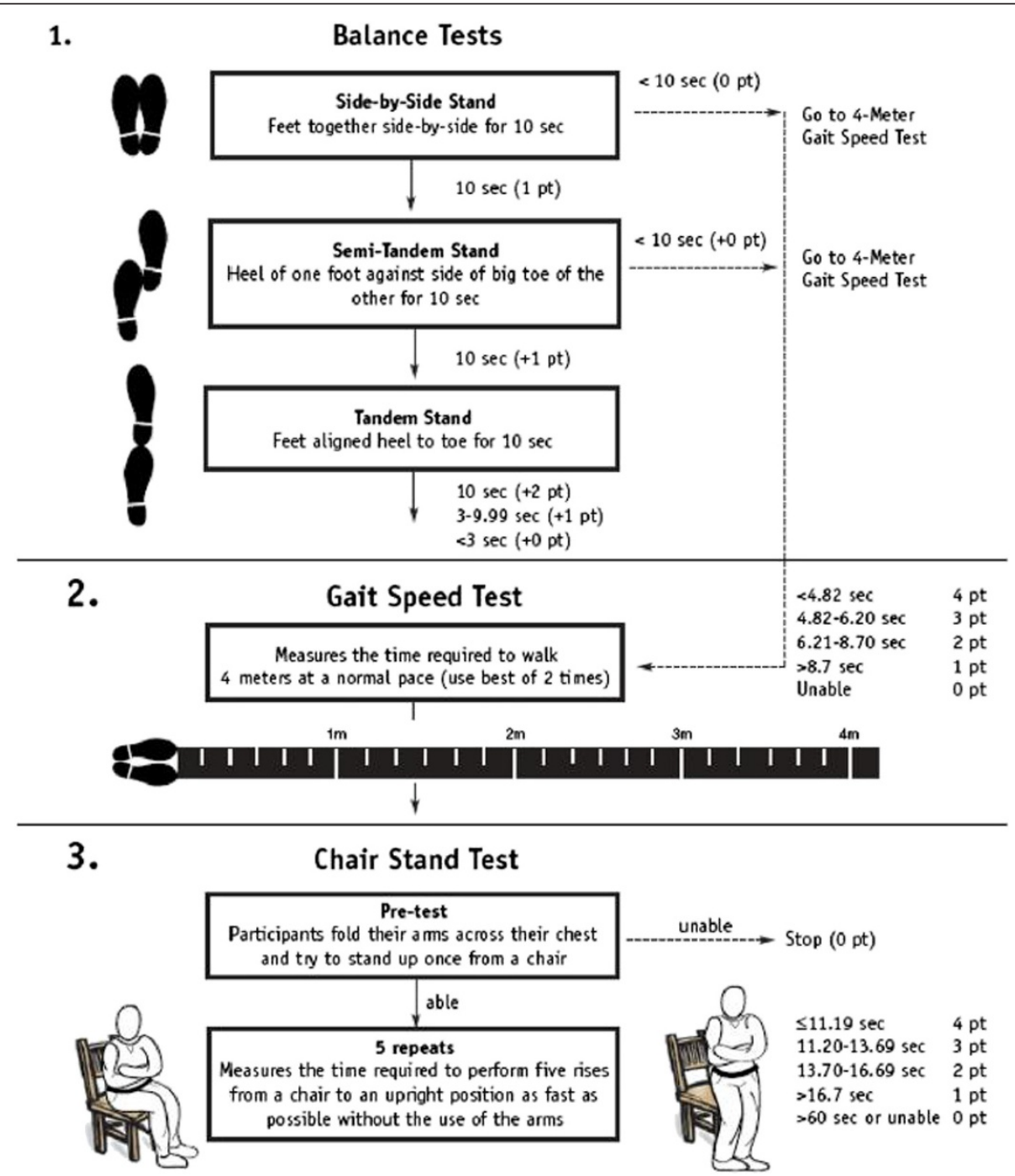

Figure 1 The short physical performance battery (SPPB) [21]. Reprinted from Journal of Biomechanics, Vol. 45, Riskowski JL, Hagedorn TJ, Dufour $\mathrm{AB}$ and Hannan MT, Functional foot symmetry and its relation to lower extremity physical performance in older adults: the Framingham Foot Study, pp. 1796-1802, Copyright 2012, with permission from Elsevier. 


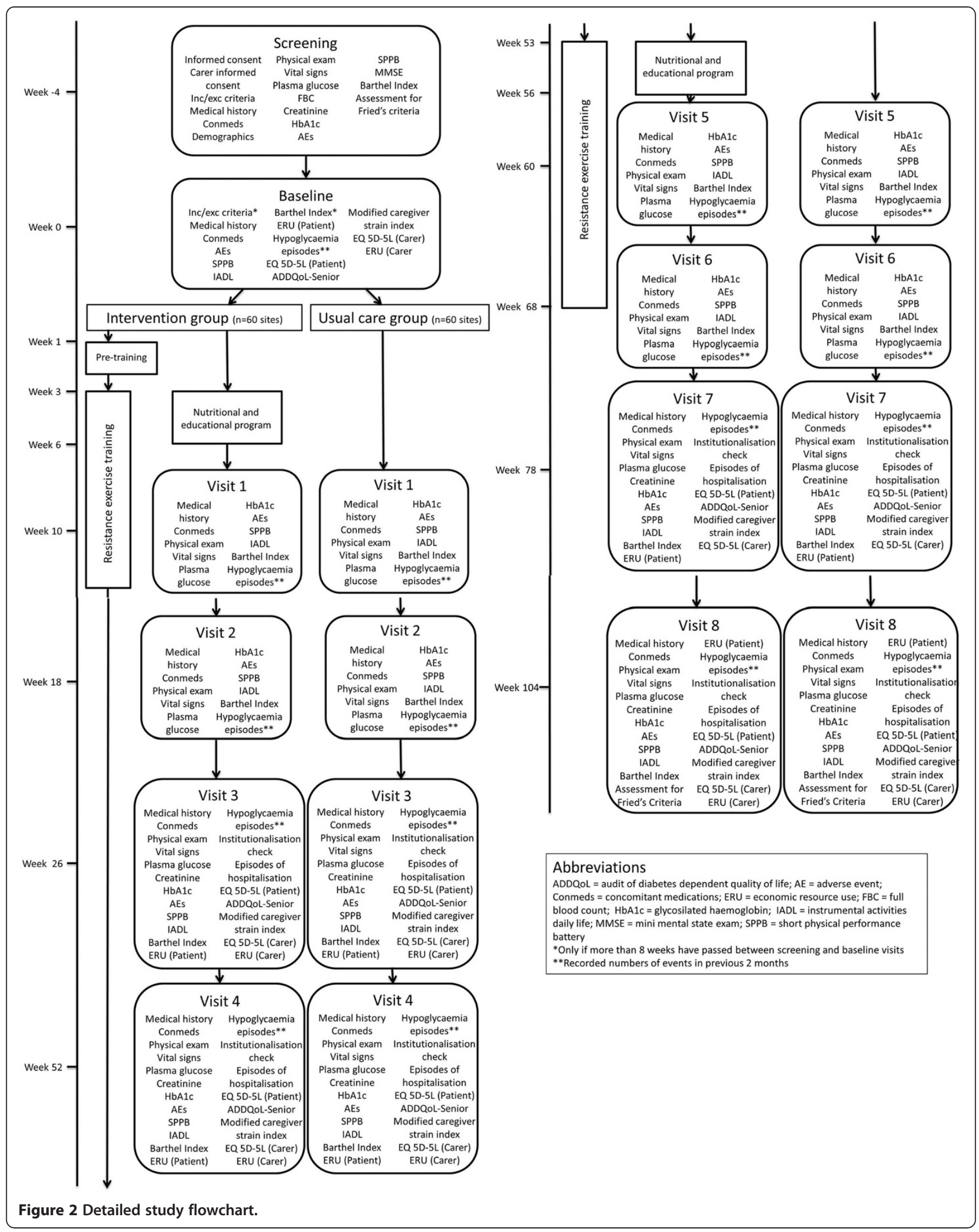


study protocol and the integrity of the data collected. An adaptive monitoring strategy has been adopted in which some sites will be monitored remotely. The study will evaluate the intervention through the difference in functioning between intervention and usual care groups; an economic evaluation of the intervention and of quality of life will also be carried out.

\section{Randomisation}

Before randomisation, each trial site will identify and make a list of 50 to 75 potential subjects. Identifying individuals for recruitment before randomisation will help to avoid subject selection bias [22]. Once this list is complete, the investigator will have 1 month to obtain signed informed consent from at least seven subjects (roughly half of the site target size) on that list. Then, the site will be randomised and exercise machines delivered. Randomisation will be performed centrally by onmedic (Barcelona, Spain) in collaboration with Cardiff University (Cardiff, UK).

\section{Intervention group}

The study intervention consists of a multi-modal programme comprising glycaemia and blood pressure monitoring to reach pre-defined targets, a 16-week resistance exercise programme based on leg press and leg extension exercises (Additional file 2), and a nutritional and educational programme. The exercise and the nutritional and educational programmes will be run at the study site at the beginning of year 1 and repeated at the beginning of year 2 . All subjects in this intervention group will follow the multi-modal programme.

Glycaemia and blood pressure monitoring The targets for glycaemia and blood pressure control will be predefined; however, the treatment required to achieve them will not be pre-defined. Treatment protocols at each site will be used to ensure that target values are reached within 6 months of enrolment. There will be no target for cholesterol or its fractions and there are no specific recommendations for the use of aspirin. The target for glycaemia will be: optimal glycated haemoglobin in the range of 7 to $8 \%$ ( 53 to $64 \mathrm{mmol} / \mathrm{mol}$ ); the target for blood pressure will be $<150 / 90 \mathrm{mmHg}$.

Exercise programme Subjects will undergo 2 weeks of pre-training assessment before the exercise programme begins. They will be familiarised with the exercises through several submaximal and maximal performances, using one-repetition maximum (1RM), and baseline measurements will be taken. In all tests of exercise performance, subjects will be encouraged verbally to perform each test action as forcefully and as rapidly as possible.
Exercise methodology The exercise programme used in the MID-Frail study will be similar to one described previously $[10,23]$. The subjects will be asked to report to the training facility on 2 days each week; each session will last from 20 to 30 minutes. Training sessions will be separated by a minimum of 2 days. Subjects will undertake supervised resistance exercise for 16 weeks starting at week 2 (baseline) and the assessments carried out at baseline will be repeated in week 6 , week 10 , and week 18. Each training session will include two exercises for the leg extensor and knee extensor muscles. Only resistance machines will be used throughout the training period (Exercycle, Exercycle S.L., Alava, Spain). For the first 8 weeks of the training period, subjects will train with loads of 40 to $60 \%$ of the individual 1RM, 8 to 12 repetitions per set, and 2 to 3 sets. For the last 8 weeks of the training period, the loads will be 70 to $80 \%$ of the maximum, 4 to 6 repetitions per set (higher loads), and 3 to 4 sets.

Nutritional and educational programme The MIDFrail nutritional and educational programme is aimed specifically at older people with diabetes and is derived from a published programme for this particular population [24]. The programme consists of sessions that aim to increase the subject's knowledge and understanding of diabetes, to develop practical skills in diabetes selfmanagement, and to enhance the likelihood of improved and safe control of glycaemia [25].

Each subject will undergo pre-trial assessment of nutritional status and any requirement for weight reduction or weight gain and the need for diet modification. Thereafter, the intervention consists of clinician-moderated sessions in a clinic or general practitioner surgery with small groups of four to eight subjects. Seven separate 45-minute sessions will be delivered, with two each week for 3 to 4 weeks; the sessions will be held on the same day as the exercise sessions.

\section{Usual care group}

Subjects in the usual care group will receive the routine care a subject with diabetes would normally be expected to receive from his/her local health care system, including his/her general practitioner.

\section{Outcome measures}

The primary outcome measure is the incidence of functional impairment as measured by the difference in function after 2 years of follow-up between intervention and usual care groups, assessed by changes in summary ordinal score on the SPPB test $\geq 1$. Data from a previous study have shown that one point is the minimum change in the SPPB test that can be considered clinically significant [26]. Secondary outcome measures are listed in Table 2. 
Table 2 Primary and secondary outcome measures

\begin{tabular}{|c|c|}
\hline Outcome measure & Definition \\
\hline Primary & $\begin{array}{l}\text { The difference in function after } 2 \text { years of follow-up between intervention and usual care groups, according to changes in } \\
\text { summary ordinal score on the SPPB test } \geq 1[26] \text {. }\end{array}$ \\
\hline \multirow[t]{8}{*}{ Secondary } & a) Barthel ADL index [13] and b) Lawton IADL scale [27]. \\
\hline & c) Quality of life, as measured by using the EuroQoL index, EQ-5D-5 L [28]. \\
\hline & $\begin{array}{l}\text { d) Economic costs/health care expenditure due to diabetes and its impact on disability and quality of life, using an economic } \\
\text { model embracing the direct health-related costs (in-subject, out-subject, pharmaceutical), formal care costs (home care, } \\
\text { respite care, day centres), and the informal care costs (carer). }\end{array}$ \\
\hline & $\begin{array}{l}\text { e) Episodes of symptomatic hypoglycaemia (that is, a recorded blood sugar }<4 \mathrm{mmol} / \mathrm{L} \text {, or symptoms or signs attributed to low } \\
\text { blood sugar and responding to appropriate treatment). }\end{array}$ \\
\hline & f) Episodes of hospital admission (that is, any admission involving an overnight stay). \\
\hline & $\begin{array}{l}\text { g) Episodes of permanent institutionalisation (that is, permanent move to any care setting other than the subject's own home, } \\
\text { where paid staff are available to provide care if needed at any time during the day or night). }\end{array}$ \\
\hline & h) Burden of the carer, as assessed by the Modified Caregiver Strain Index (MCSI) [29]. \\
\hline & i) Mortality. \\
\hline
\end{tabular}

ADL, activities of daily living; IADL, instrumental activities of daily living; MCSI, Modified Caregiver Strain Index; SPPB, short physical performance battery.

\section{Economic assessments}

The main aim of the economic assessment is to estimate the incremental cost-effectiveness ratio of the multi-modal intervention in frail and pre-frail subjects aged $\geq 70$ years with type 2 diabetes in comparison with usual best clinical practice. Three types of cost will be assessed through a comparative analysis of the alternative courses of action in terms of both their financial costs and their health outcomes: 1) direct health care costs, these costs are related to in-subject and out-subject treatment, and medicines; 2) formal care (mainly social services) costs, this includes those services that involve public or private funding, including the use of a day centre, nursing home, residential care, home support services, and personal alarm system; and 3) informal care costs, informal support includes the different types of non-paid support provided by relatives and friends as a result of the subject's disability.

\section{Safety assessments}

All co-existing diseases or conditions will be treated in accordance with prevailing medical practice. All medications (prescription and over-the-counter) that started before screening may be continued during the study and will be recorded as concomitant therapy on the electronic case report form (eCRF). Medications for any conditions that may arise after screening, or for worsening of an existing condition, will be allowed and recorded on the eCRF; the condition will be reported as an adverse event. Standard safety data on adverse drug reactions will be collected and reported.

\section{Power and sample size}

The sample size per group (intervention and usual care group) will be 859 subjects. This sample size has been calculated according to the following assumptions: a yearly incidence of functional impairment in frail/pre-frail subjects (main variable) of 30\% [30], accumulated incidence of functional impairment in 2 years of intervention of $51 \%$ (assuming a constant 30\% incidence rate year on year), an intervention effect size of $20 \%$, a z statistic to compare the proportions of dichotomous variables (as the statistical test), a two-tailed $\alpha=0.05$, a two-tailed $1-\beta=0.8$, an intracluster coefficient correlation of 0.05 , an average cluster size of 15 , and a coefficient of variation cluster size of 0.25 $[31,32]$. Assuming a $20 \%$ loss of subjects during follow-up, the final sample size will be 1,718 subjects.

\section{Statistical analysis}

The primary analysis will compare the odds of developing functional impairment in the intervention group with the usual care group, controlling for baseline function, using a hierarchical logistic regression model with subjects nested within trial sites. This will be an intention-to-treat analysis controlling for subject characteristics, such as subject age, gender, and comorbidities. The groups will be compared in terms of odds ratios and relative risks.

Secondary outcome variables include: 1) quality of life as measured by the quality of life measurement tool EQ5D-5 L (EuroQoL, Rotterdam, Netherlands), which will be used in the economic evaluation; 2) hospital admission, mortality, and permanent institutionalisation, which will be investigated using hierarchical logistic regression models, and the results summarised using odds ratios and relative risks. If there are multiple outcomes per subject (for example hospital admission), Poisson regression may be used. Hierarchical survival analysis may also be used to model the time to first event; 3) carer burden and quality of life, which will be addressed using hierarchical regression models; and 4) validation of the Audit of Diabetes-Dependent Quality of Life (ADDQoL) senior scale [33], including measuring internal consistency (using Cronbach's alpha) and establishing convergent and 
divergent validity (by comparing subject characteristics expected and not expected to be related with ADDQoL senior and ADDQoL senior scores). Floor and ceiling effects will be investigated. Correlation analysis will be conducted to identify whether the ADDQoL senior scale correlates with the other measures of function used in this study. Test re-test reliability will also be estimated (the ADDQoL senior will be administered at weeks $0,26,52$, 78 , and 104).

All analyses, except for the economic assessment, will be run by team members at Cardiff University in collaboration with the study and sub-study teams. The economic analysis will be performed by team members from Universidad Castilla-La Mancha (Ciudad Real, Spain). Standard imputation methods (for example mean value imputation, last observation carried forward) will be used to impute missing data depending on the pattern of missing data. Dropout due to death is a possibility in this study population. A sensitivity analysis will be run in which dropouts due to death will be assigned the minimum function score. Joint modelling of function and dropout due to death will also be explored. A causal adjusted complier analysis will be used to estimate the treatment effect observed in those subjects who complied with the intervention. Survival analysis naturally deals with censoring, which will help minimise the effect of dropout in the secondary analyses. No interim analysis is planned and is not accounted for in the sample size calculation. An ethics independent external advisory committee will be formed and will meet at least three times during the course of the study to evaluate its progress, the safety data, the critical efficacy endpoints, and will make any recommendations to the sponsor and the steering committee whether to continue, modify, or stop the study.

\section{Discussion}

The rapid increase in the number of older people combined with the high prevalence of diabetes in ageing populations has created an urgent need for effective interventions to prevent or delay the onset of frailty and functional decline in older people. The MID-Frail study will address the lack of intervention studies in older people with diabetes by examining a comprehensive, multi-modal intervention designed to reduce functional decline and improve quality of life.

It is estimated that if the MID-Frail intervention is successful in reducing disability and functional decline, 700,000 fewer cases of disability will be reported every year, accompanied by health care savings of more than 3 billion euros per year across the EU. The MID-Frail nutritional and educational programme is intended to prevent loss of muscle protein and ensure optimal nutritional status, to minimise the risk of hypoglycaemia, and assist in maintaining functional status. Investigators will be guided on how to prepare each session and how to maximise participants' enjoyment and benefits through interaction. Individual nutritional goals will be set for each subject and there will be a focus on behavioural change. The specific dietary needs of older people will be emphasised and the programme will be tailored in each country to take into account the influence local social and cultural norms have on diet.

The study will be randomised using the cluster method; this is necessary because the education and the exercise programmes will be done in groups, and this will also avoid or control for contamination bias. The MID-Frail study will run for 4 years with a 2 -year intervention. We will capitalise on the long duration of the study and the large number of subjects involved by carrying out several sub-studies, which will run concurrently with the main study and will evaluate complementary research questions: 1) the characteristics of skeletal muscle and adjacent tissues will be investigated by sonoelastography at the beginning and at the end of the intervention (Sartrain Substudy); 2) exercise-induced, short- and long-term changes in muscle power output, balance, and gait will be studied as mediators of the final response on function (MID-POW Sub-study); 3) kinetic and kinematic movement data will be analysed (Sensole Sub-study); 4) the metabolic profile of frail/sarcopenic older subjects with diabetes will be characterised and changes in these parameters following the intervention will be profiled (MetaboFrail Sub-study); and 5) polymorphisms of three genes (Pro259Arg-TCN2 gene; ACE I/D-ACE gene, and e2/e3/e4-Apo $E$ gene) will be determined to establish their predictive value for the development of disability and response to treatment (GeneFrail Sub-study).

The MID-Frail study will provide evidence on the clinical, functional, social, and economic impact of a multimodal approach in frail and pre-frail older people with diabetes.

\section{Trial status}

The trial is currently being set up.

\section{Additional files}

Additional file 1: Details of institutional ethics committees.

Additional file 2: Leg exercises.

\section{Abbreviations}

1RM: One-repetition maximum; ADDQoL: Audit of Diabetes-Dependent Quality of Life; ADL: Activities of daily living; BMI: Body mass index; CES-D: Center for Epidemiologic Studies Depression; eCRF: Electronic case report form; EU: European Union; HDL: High-density lipoprotein; IADL: Instrumental activities of daily living; MCSI: Modified Caregiver Strain Index; MIDFrail: Multi-modal intervention in diabetes in frailty; MMSE: Mini Mental State Examination; NYHA: New York Heart Association; SPPB: Short physical performance battery. 


\section{Competing interests}

The authors declare that they have no competing interests.

\section{Authors' contributions}

LRM participated in the conception, design, and writing of the study. AJB outlined the statistical analysis and performed the sample size calculations. MK provided input on the statistical analysis. AZ designed the educational intervention. MI designed the exercise programme. OL participated in the design of the study. TCH participated in the design and writing of the study. AJS participated in the conception, design, and writing of the study. All authors read and approved the final manuscript.

\section{Acknowledgements}

The authors thank the University of UIm (UIm, Germany) for helping with administrative tasks. The MID-Frail consortium comprises the following organisations: Hospital Universitario de Getafe (Getafe, Spain); Institute of Diabetes for Older People (Luton, UK); Cardiff University (Cardiff, UK); Igen Biotech (Madrid, Spain); Centre Hospitalier Universitaire de Bordeaux (Bordeaux, France); HEXABIO, University of Naples (Naples, Italy); Hôpital La Grave-Casselardit (Toulouse, France); Agaplesion Bethesda Krankenhaus (Stuttgart, Germany); Ghent University (Ghent, Belgium); Universidad de Castilla-La Mancha (Ciudad Real, Spain); Charles University (Prague, Czech Republic); Consorcio de Apoyo a la Investigación Biomédica en Red (CAIBER, Madrid, Spain; participation withdrawn in June 2012); Niche Science and Technology (Richmond-upon-Thames, UK); Centro Medicinadell'Invecchiamento, Università Cattolica Sacro Cuore (Rome, Italy); and Vrije Universiteit Brussel (Brussels, Belgium). This study was funded by the European Commission Seventh Framework Programme. Dr Severina Moreira and Dr Justin Cook from Niche Science and Technology, a member of the MID-Frail Study consortium, provided writing and editorial support for the development of this manuscript.

\section{Author details}

'Division of Geriatrics, Hospital Universitario de Getafe, Carretera de Toledo Km 12.5, 28905, Getafe, Spain. ²Fundación para la Investigación Biomédica, Hospital Universitario de Getafe, Carretera de Toledo Km 12.5 28905, Getafe, Spain. ${ }^{3}$ Institute of Primary Care and Public Health, School of Medicine, Cardiff University and Memory Team, Cardiff and Vale University Health Board, Cardiff CF14 4YS, UK. Institute of Translation, Innovation, Methodology and Engagement (TIME), South East Wales Trials Unit, School of Medicine, Cardiff University, Cardiff CF14 4XN, UK. ${ }^{5}$ University of Ulm, Institue of Epidemiology, Albert-Einstein-Allee 41, 89081 Ulm, Germany. ${ }^{6}$ Department of Health Sciences, Public University of Navarre, Campus of Tudela 31500 Tudela, Spain. ${ }^{7}$ Niche Science \& Technology, Unit 26, Falstaff House, Bardolph Road, Richmond, TW9 2LH, UK. ${ }^{8}$ Institute of Diabetes for Older People (IDOP), University of Bedfordshire, Putteridge Bury Campus, Hitchin Road, Luton, Bedfordshire LU2 8LE, UK.

Received: 24 April 2013 Accepted: 11 December 2013 Published: 24 January 2014

\section{References}

1. Sinclair AJ: Towards a minimum data set for intervention studies in type 2 diabetes in older people. J Nutr Health Aging 2007, 11:289-293.

2. Sinclair AJ, Conroy SP, Bayer AJ: Impact of diabetes on physical function in older people. Diabetes Care 2008, 31:233-235.

3. Fried LP, Tangen CM, Walston J, Newman AB, Hirsch C, Gottdiener J, Seeman T, Tracy R, Kop WJ, Burke G, McBurnie MA, Cardiovascular Health Study Collaborative Research Group: Frailty in older adults: evidence for a phenotype. J Gerontol A Biol Sci Med Sci 2001, 56:M146-M156.

4. Oliva J, Lobo F, Molina B, Monereo S: Direct health care costs of diabetic patients in Spain. Diabetes Care 2004, 27:2616-2621.

5. Volpato S, Maraldi C, Fellin R: Type 2 diabetes and risk for functional decline and disability in older persons. Curr Diabetes Rev 2010, 6:134-143.

6. Lu F-P, Lin K-P, Kuo H-K: Diabetes and the risk of multi-system aging phenotypes: a systematic review and meta-analysis. PLoS One 2009, 4:e4144.

7. Laiteerapong N, Karter AJ, Liu JY, Moffet HH, Sudore R, Schillinger D, John PM, Huang ES: Correlates of quality of life in older adults with diabetes: the diabetes \& aging study. Diabetes Care 2011, 34:1749-1753.

8. Rosso AL, Eaton CB, Wallace R, Gold R, Curb JD, Stefanick ML, Ockene JK, Michael YL: Combined impact of geriatric syndromes and cardiometabolic diseases on measures of functional impairment. J Gerontol A Biol Sci Med Sci 2011, 66A:349-354.

9. Rodriguez-Manas $\mathrm{L}$ : A call for action in diabetes care for older people. Diabet Med 2011, 28:757-758.

10. Ibañez J, Izquierdo M, Argüelles I, Forga L, Larrión JL, García-Unciti M, Idoate F, Gorostiaga EM: Twice-weekly progressive resistance training decreases abdominal fat and improves insulin sensitivity in older men with type 2 diabetes. Diabetes Care 2005, 28:662-667.

11. Cadore EL, Rodríguez-Mañas L, Sinclair A, Izquierdo M: Effects of different exercise interventions on risk of falls, gait ability and balance in physically frail older adults: a systematic review. Rejuvenation Res 2013, 16:105-114.

12. Action to Control Cardiovascular Risk in Diabetes Study Group, Gerstein HC, Miller ME, Byington RP, Goff DC Jr, Bigger JT, Buse JB, Cushman WC, Genuth S, Ismail-Beigi F, Grimm RH Jr, Probstfield JL, Simons-Morton DG, Friedewald WT: Effects of intensive glucose lowering in type 2 diabetes. N Engl J Med 2008, 358:2545-2559.

13. ACCORD Study Group, Gerstein HC, Miller ME, Genuth S, Ismail-Beigi F, Buse JB, Goff DC Jr, Probstfield JL, Cushman WC, Ginsberg HN, Bigger JT, Grimm $\mathrm{RH}$ Jr, Byington RP, Rosenberg YD, Friedewald WT: Long-term effects of intensive glucose lowering on cardiovascular outcomes. N Engl J Med 2011, 364:818-828.

14. Look AHEAD Research Group, Wing RR: Long-term effects of a lifestyle intervention on weight and cardiovascular risk factors in individuals with type 2 diabetes mellitus: four-year results of the look AHEAD trial. Arch Intern Med 2010, 170:1566-1575.

15. Look AHEAD Research Group, Wing RR, Bolin P, Brancati FL, Bray GA, Clark JM, Coday M, Crow RS, Curtis JM, Egan CM, Espeland MA, Evans M, Foreyt JP, Ghazarian S, Gregg EW, Harrison B, Hazuda HP, Hill JO, Horton ES, Hubbard VS, Jakicic JM, Jeffery RW, Johnson KC, Kahn SE, Kitabchi AE, Knowler WC, Lewis CE, Maschak-Carey BJ, Montez MG, Murillo A, et al: Cardiovascular effects of intensive lifestyle intervention in type 2 diabetes. N Engl J Med 2013, 369:145-154.

16. MID-Frail. http://www.midfrail-study.org.

17. Mahoney FI, Barthel DW: Functional evaluation: the barthel index. Md State Med J 1965, 14:61-65.

18. Guralnik JM, Ferrucci L, Simonsick EM, Salive ME, Wallace RB: Lowerextremity function in persons over the age of 70 years as a predictor of subsequent disability. N Engl J Med 1995, 332:556-561.

19. Folstein MF, Folstein S, McHugh PR: "Mini-mental state": a practical method for grading the cognitive state of patients for the clinician. J Psych Res 1975, 12:189-198.

20. The Criteria Committee of the New York Heart Association: Nomenclature and Criteria for Diagnosis of Diseases of the Heart and Great Vessels. 9th edition. Boston, MA: Little, Brown \& Co; 1994:253-256.

21. Riskowski JL, Hagedorn TJ, Dufour AB, Hannan MT: Functional foot symmetry and its relation to lower extremity physical performance in older adults: the Framingham foot study. J Biomech 2012, 45:1796-1802.

22. Hahn S, Puffer S, Torgerson DJ, Watson J: Methodological bias in cluster randomised trials. BMC Med Res Methodol 2005, 5:10.

23. Izquierdo M, Häkkinen K, Ibañez J, Garrues M, Antón A, Zúñiga A, Larrión JL, Gorostiaga EM: Effects of strength training on muscle power and serum hormones in middle-aged and older men. J App/ Physio/ 2001, 90:1497-1507.

24. Braun AK, Kubiak T, Kuntsche J, Meier-Höfig M, Müller UA, Feucht I, Zeyfang A: SGS: a structured treatment and teaching programme for older patients with diabetes mellitus - a prospective randomised controlled multi-centre trial. Age Ageing 2009, 38:390-396.

25. Zeyfang A, Berndt S, Aurnhammer G, Nikolaus T, Oster P, Bahrmann A: A short easy test can detect ability for autonomous insulin injection by the elderly with diabetes mellitus. J Am Med Dir Assoc 2012, 13:81. e15-18.

26. Kwon S, Perera S, Pahor M, Katula JA, King AC, Groessl EJ, Studenski SA: What is a meaningful change in physical performance? findings from a clinical trial in older adults (the LIFE-P study). J Nutr Health Aging 2009, 13:538-544.

27. Lawton MP, Brody EM: Assessment of older people: self-maintaining and instrumental activities of daily living. Gerontologist 1969, 9:179-186.

28. EuroQol Group. http://www.euroqol.org.

29. Sinclair AJ, Armes DG, Randhawa G, Bayer AJ: Caring for older adults with diabetes mellitus: characteristics of carers and their prime roles and responsibilities. Diabet Med 2010, 27:1055-1059.

30. LIFE Study Investigators, Pahor M, Blair SN, Espeland M, Fielding R, Gill TM, Guralnik JM, Hadley EC, King AC, Kritchevsky SB, Maraldi C, Miller ME, 
Newman AB, Rejeski WJ, Romashkan S, Studenski S: Effects of a physical activity intervention on measures of physical performance: results of the lifestyle interventions and independence for Elders Pilot (LIFE-P) study. J Gerontol A Biol Sci Med Sci 2006, 61:1157-1165.

31. Elridge $S M$, Ashby D, Kerry S: Sample size for cluster randomized trials: effect of coefficient of variation of cluster size and analysis method. Int $\rfloor$ Epidemiol 2006, 35:1292-1300.

32. Voigt-Radloff S, Schochat T, Heiss HW: Controlled trials on the efficacy of occupational therapy with elderly: part I: research question, search strategy and methodological quality of trials. Z Gerontol Geriatr 2004, 37:444-449

33. Speight J, Sinclair AJ, Browne JL, Woodcock A, Bradley C: Assessing the impact of diabetes on the quality of life of older adults living in a care home: validation of the ADDQoL Senior. Diabet Med 2013, 30:74-80.

doi:10.1186/1745-6215-15-34

Cite this article as: Rodríguez-Mañas et al:: An evaluation of the

effectiveness of a multi-modal intervention in frail and pre-frail older people with type 2 diabetes - the MID-Frail study: study protocol for a randomised controlled trial. Trials 2014 15:34.

\section{Submit your next manuscript to BioMed Central and take full advantage of:}

- Convenient online submission

- Thorough peer review

- No space constraints or color figure charges

- Immediate publication on acceptance

- Inclusion in PubMed, CAS, Scopus and Google Scholar

- Research which is freely available for redistribution 